\title{
TORIC KÄHLER METRICS: COHOMOGENEITY ONE EXAMPLES OF CONSTANT SCALAR CURVATURE IN ACTION-ANGLE COORDINATES
}

\author{
MIGUEL ABREU
}

\begin{abstract}
In these notes, after an introduction to toric Kähler geometry, we present Calabi's family of $U(n)$-invariant extremal Kähler metrics in symplectic action-angle coordinates and show that it actually contains, as particular cases, many interesting cohomogeneity one examples of constant scalar curvature.
\end{abstract}

\section{INTRODUCTION}

In 1982 Calabi [7] constructed, in local complex coordinates, a general 4-parameter family of $U(n)$-invariant extremal Kähler metrics, which he used to put an extremal Kähler metric on

$$
H_{m}^{n}:=\mathbb{P}(\mathcal{O}(-m) \oplus \mathbb{C}) \rightarrow \mathbb{P}^{n-1},
$$

for all $n, m \in \mathbb{N}$ and any possible Kähler cohomology class. In particular, when $n=2$, on all Hirzebruch surfaces.

The main goal of these notes is to present Calabi's general family in local symplectic actionangle coordinates, using the set-up of [1, 2] for toric Kähler geometry, and show that it actually contains other interesting cohomogeneity one Kähler metrics as particular cases (see also [22]). These include:

- the Fubini-Study, flat and Bergman Kähler-Einstein metrics of constant holomorphic sectional curvature (positive, zero and negative, resp.).

- the complete Ricci flat Kähler metric on the total space of

$$
\mathcal{O}(-n) \rightarrow \mathbb{P}^{n-1},
$$

for all $n \in \mathbb{N}$ and any possible Kähler cohomology class, constructed by Calabi [6] in 1979 .

- the complete scalar flat Kähler metric on the total space of

$$
\mathcal{O}(-m) \rightarrow \mathbb{P}^{n-1}
$$

for all $m, n \in \mathbb{N}$ and any possible Kähler cohomology class, constructed for $n=2$ by LeBrun [18] in 1988 and for $n>2$ by Pedersen-Poon [21] in 1991 (see also Simanca [24]).

- the complete Kähler-Einstein metric with negative scalar curvature on the total space of the open disc bundle

$$
\mathcal{D}(-m) \subset \mathcal{O}(-m) \longrightarrow \mathbb{P}^{n-1},
$$

for all $n<m \in \mathbb{N}$ and any possible Kähler cohomology class, constructed by PedersenPoon [21].

- the complete constant negative scalar curvature Kähler metric on the total space of the open disc bundle

$$
\mathcal{D}(-m) \subset \mathcal{O}(-m) \longrightarrow \mathbb{P}^{n-1},
$$

for all $n, m \in \mathbb{N}$ and any possible Kähler cohomology class, also constructed by PedersenPoon [21].

Calabi's general family contains many other interesting cohomogeneity one special Kähler metrics. Besides the Bochner-Kähler orbifold examples presented in [3], it contains for example a family of singular Kähler-Einstein metrics on certain $H_{m}^{n}$ that are directly related to the SasakiEinstein metrics constructed by Gauntlett-Martelli-Sparks-Waldram [13, 14] in 2004 - see [4].

Partially supported by the Fundação para a Ciência e a Tecnologia (FCT/Portugal). 
These notes are organized as follows. In section 2 we give a basic introduction to symplectic geometry and discuss some fundamental features of toric symplectic manifolds. Section 3 is devoted to toric Kähler metrics. After some relevant linear algebra prelimaries, we explain how these can be parametrized in action-angle coordinates via symplectic potentials for the associated toric compatible complex structures, and discuss some important properties of these symplectic potentials. In section 4 we write down the symplectic potentials that give rise to toric constant (scalar) curvature metrics in real dimension 2 and identify the underlying toric symplectic surfaces. This is a warm-up for section 5, where we discuss in detail the above higher dimensional examples that arise in Calabi's general family of local $U(n)$-invariant extremal Kähler metrics.

Acknowledgements. I thank the organizers of the XI International Conference on Geometry, Integrability and Quantization, Varna, Bulgaria, June 5-10, 2009, where this material was presented as part of a mini-course.

The work presented in Section 5 was carried out in January-June of 2001, while I was at the Fields Institute for Research in Mathematical Sciences, Toronto, Canada. It was presented in seminar talks given at the Workshop on Hamiltonian Group Actions and Quantization, Fields Institute, June 4-13, 2001, and at Differentialgeometrie im Großen, Mathematisches Forschungsinsitut Oberwolfach, Germany, June 10-16, 2001. I thank the support and hospitality of the Fields Institute and the organizers of those meetings.

\section{Toric Symplectic Manifolds}

In this section we give a basic introduction to symplectic geometry and discuss some fundamental features of toric symplectic manifolds.

\section{Symplectic Manifolds.}

Definition 2.1. A symplectic manifold is a pair $(B, \omega)$ where $B$ is a smooth manifold and $\omega$ is a closed and non-degenerate 2-form, i.e.

(i) $\omega \in \Omega^{2}(B)$ is such that $d \omega=0$ and

(ii) for any $p \in B$ and $0 \neq X \in T_{p} B$, there exists $Y \in T_{p} B$ such that $\omega_{p}(X, Y) \neq 0$.

The non-degeneracy condition (ii) implies that a symplectic manifold is always even dimensional. If $B$ has dimension $2 n$, the non-degeneracy condition (ii) is equivalent to requiring that

$$
\omega^{n} \equiv \omega \wedge \cdots \wedge \omega \in \Omega^{2 n}(B) \text { is a volume form. }
$$

Hence, a symplectic manifold $(B, \omega)$ is always oriented.

Example 2.2. The most basic example is $\mathbb{R}^{2 n}$ with linear coordinates

$$
\left(u_{1}, \ldots, u_{n}, v_{1}, \ldots, v_{n}\right)
$$

and symplectic form

$$
\omega_{\mathrm{st}}=d u \wedge d v:=\sum_{j=1}^{n} d u_{j} \wedge d v_{j} .
$$

Example 2.3. Any 2-dimensional surface equipped with an area form is a symplectic manifold. For example, the sphere $S^{2}$ or any other compact orientable surface $\Sigma_{g}$ of genus $g$.

Example 2.4. If $\left(B_{1}, \omega_{1}\right)$ and $\left(B_{2}, \omega_{2}\right)$ are symplectic manifolds, then

$$
\left(B=B_{1} \times B_{2}, \omega=\omega_{1} \times \omega_{2}\right)
$$

is also a symplectic manifold. Here, $\omega_{1} \times \omega_{2}$ means the sum of the pullbacks of the symplectic forms $\omega_{1}$ and $\omega_{2}$ from the factors $B_{1}$ and $B_{2}$.

Example 2.5. The imaginary part of the hermitean metric on any Kähler manifold is a symplectic form. Hence, any Kähler manifold is a symplectic manifold. In particular, the complex projective space $\mathbb{P}^{n}$ equipped with its Fubini-Study form $\omega_{F S}$ is a symplectic manifold. 
When $(B, \omega)$ is a compact symplectic manifold we have that

$$
\omega^{n}=\text { volume form } \Rightarrow 0 \neq\left[\omega^{n}\right] \in H^{2 n}(B, \mathbb{R}) \Rightarrow 0 \neq[\omega] \in H^{2}(B, \mathbb{R}) .
$$

In particular, the spheres $\mathbb{S}^{2 n}$ have no symplectic form when $n>1$, since

$$
H^{2}\left(\mathbb{S}^{2 n}, \mathbb{R}\right)=0 \quad \text { when } n>1 .
$$

\section{Symplectomorphisms and Darboux's Theorem.}

Definition 2.6. Let $(B, \omega)$ be a symplectic manifold. A symplectomorphism of $B$ is a diffeomorphism $\varphi: B \rightarrow B$ such that $\varphi^{*}(\omega)=\omega$. These form the symplectomorphism group, a subgroup of $\operatorname{Diff}(B)$ that will be denoted by $\operatorname{Diff}(B, \omega)$.

Example 2.7. Consider a symplectic manifold $(B, \omega)$ and let $h: B \rightarrow \mathbb{R}$ be a smooth function on $B$. The non-degeneracy of $\omega$ implies that there exists a unique vector field $X_{h} \in \mathcal{X}(B)$ such that $\left.X_{h}\right\lrcorner \omega=d h$. This vector field $X_{h}$ is called the Hamiltonian vector field of the function $h$ and has the following fundamental property:

$$
\text { the flow } \varphi_{t} \equiv\left(X_{h}\right)_{t}: B \rightarrow B \text { consists of symplectomorphisms of } B \text {. }
$$

This can be proved using Cartan's formula to compute

$$
\left.\left.\left.\mathcal{L}_{X_{h}} \omega=X_{h}\right\lrcorner d \omega+d\left(X_{h}\right\lrcorner \omega\right)=X_{h}\right\lrcorner 0+d(d h)=0 .
$$

Hence, on a symplectic manifold $(B, \omega)$ any smooth function $h \in C^{\infty}(B)$ gives rise, through the flow of the corresponding Hamiltonian vector field $X_{h} \in \mathcal{X}(B)$, to a 1-parameter group of symplectomorphisms.

One can use the symplectomorphisms constructed in the previous example to prove that:

(i) the symplectomorphism group $\operatorname{Diff}(B, \omega)$ is always infinite-dimensional;

(ii) the action of $\operatorname{Diff}(B, \omega)$ on the manifold $B$ is always $k$-transitive, for any $k \in \mathbb{N}$;

(iii) in particular, any point of a symplectic manifold $(B, \omega)$ looks locally like any other point of $(B, \omega)$.

This last statement is made more precise in the following

Theorem 2.8. [Darboux] Let $(B, \omega)$ be a symplectic manifold of dimension $2 n$. Then, any point $p \in B$ has a neighborhood $U \subset B$ symplectomorphic to a neighborhood $V$ of the origin in $\left(\mathbb{R}^{2 n}, \omega_{\mathrm{st}}\right)$, i.e. there exists a diffeomorphism

$$
\phi: U \subset B \rightarrow V \subset \mathbb{R}^{2 n} \text { such that } \phi(p)=0 \text { and } \phi^{*}\left(\omega_{\mathrm{st}}\right)=\omega .
$$

In other words,

there are no local invariants in symplectic geometry,

which is in sharp contrast with what happens, for example, in Riemannian geometry.

Symplectic and Hamiltonian Vector Fields. The Lie algebra of the symplectomorphism group $\operatorname{Diff}(B, \omega)$, viewed as an infinite-dimensional Lie group, is naturally identified with the vector space $\mathcal{X}(B, \omega)$ of symplectic vector fields, i.e. vector fields $X \in \mathcal{X}(B)$ such that $\mathcal{L}_{X} \omega=0$, with Lie bracket $[\cdot, \cdot]$ given by the usual Lie bracket of vector fields. As before, we can use Cartan's formula to obtain

$$
\left.\left.\left.\left.\left.\mathcal{L}_{X} \omega=X\right\lrcorner d \omega+d(X\lrcorner \omega\right)=X\right\lrcorner 0+d(X\lrcorner \omega\right)=d(X\lrcorner \omega\right) .
$$

Hence, the vector space of symplectic vector fields is given by

$$
\mathcal{X}(B, \omega)=\{X \in \mathcal{X}(B) \text { : the } 1 \text {-form } X\lrcorner \omega \text { is closed }\},
$$

while its subspace of Hamiltonian vector fields is given by

$$
\left.\mathcal{X}_{H}(B, \omega)=\{X \in \mathcal{X}(B) \text { : the } 1 \text {-form } X\lrcorner \omega \text { is exact }\right\} .
$$

In fact, as the following theorem shows, $\mathcal{X}_{H}(B, \omega)$ is a Lie subalgebra of $\mathcal{X}(B, \omega)$. 
Theorem 2.9. If $X, Y \in \mathcal{X}(B, \omega)$ are symplectic vector fields, then $[X, Y]$ is the Hamiltonian vector field of the function $\omega(Y, X): B \rightarrow \mathbb{R}$, i.e.

$$
[X, Y]=X_{\omega(Y, X)} \in \mathcal{X}_{H}(B, \omega) .
$$

Proof. It suffices to compute $[X, Y]\lrcorner \omega$, using standard formulas from differential geometry and the defining properties of $X, Y$ and $\omega$ :

$$
\begin{aligned}
{[X, Y]\lrcorner \omega } & \left.\left.=\mathcal{L}_{X}(Y\lrcorner \omega\right)-Y\right\lrcorner\left(\mathcal{L}_{X} \omega\right) \\
& =d(X\lrcorner(Y\lrcorner \omega))+X\lrcorner(d(Y\lrcorner \omega))-Y\lrcorner(d(X\lrcorner \omega))-Y\lrcorner(X\lrcorner d \omega) \\
& =d(\omega(Y, X)) .
\end{aligned}
$$

Remark 2.10. $\mathcal{X}_{H}(B, \omega)$ is the Lie algebra of a fundamental subgroup of the symplectomorphism group: the subgroup $\operatorname{Ham}(B, \omega) \subset \operatorname{Diff}(B, \omega)$ of Hamiltonian symplectomorphisms of $(B, \omega)$. It follows from Theorem 2.9 that this Lie algebra can be naturally identified with the vector space $C^{\infty}(B) / \mathbb{R}$, i.e. smooth functions on $B$ modulo constants, equipped with a bracket $\{\cdot, \cdot\}$ known as the Poisson bracket:

$$
\{f, g\} \equiv \omega\left(X_{f}, X_{g}\right) .
$$

Note that when $H^{1}(B, \mathbb{R})=0$ we have that $\mathcal{X}_{H}(B, \omega)=\mathcal{X}(B, \omega)$.

Hamiltonian Torus Actions. Let $(B, \omega)$ be a symplectic manifold equipped with a symplectic action of

$$
\mathbb{T}^{m} \equiv \mathbb{R}^{m} / 2 \pi \mathbb{Z}^{m} \equiv \mathbb{R} / 2 \pi \mathbb{Z} \times \cdots \times \mathbb{R} / 2 \pi \mathbb{Z} \equiv \mathbb{S}^{1} \times \cdots \times \mathbb{S}^{1}
$$

i.e. with a homomorphism $\mathbb{T}^{m} \rightarrow \operatorname{Diff}(B, \omega)$. Let $X_{1}, \ldots, X_{m} \in \mathcal{X}(B)$ be the vector fields generating the action of each individual $\mathbb{S}^{1}$-factor. Then, since the action is symplectic, we have that

$$
\left.\left.\left.\mathcal{L}_{X_{k}} \omega=0 \Leftrightarrow X_{k}\right\lrcorner d \omega+d\left(X_{k}\right\lrcorner \omega\right)=0 \Leftrightarrow d\left(X_{k}\right\lrcorner \omega\right)=0
$$

i.e.

$$
X_{k} \in \mathcal{X}(B, \omega), \forall k \in\{1, \ldots, m\}
$$

Definition 2.11. A symplectic $\mathbb{T}^{m}$-action on a symplectic manifold $(B, \omega)$ is said to be Hamiltonian if for every $k \in\{1, \ldots, m\}$ there exists a function $h_{k}: B \rightarrow \mathbb{R}$ such that $\left.X_{k}\right\lrcorner \omega=d h_{k}$, i.e. $X_{k} \equiv X_{h_{k}} \in \mathcal{X}_{H}(B, \omega)$ is the Hamiltonian vector field of $h_{k}$. In this case, the map $\mu: B \rightarrow \mathbb{R}^{m}$ defined by

$$
\mu(p)=\left(h_{1}(p), \ldots, h_{m}(p)\right), \forall p \in B,
$$

is called a moment map for the action.

Remark 2.12. Suppose $\mu: B \rightarrow \mathbb{R}^{m}$ is a moment map for a Hamiltonian $\mathbb{T}^{m}$-action on $(B, \omega)$. Then $\mu+c$, for any given constant $c \in \mathbb{R}^{m}$, is also a moment map for that same action.

Remark 2.13. The orbits of a Hamiltonian $\mathbb{T}^{m}$-action on a symplectic manifold $(B, \omega)$ are always isotropic, i.e.

$$
\left.\omega\right|_{\text {orbit }} \equiv 0 \text {. }
$$

In fact, the tangent space to an orbit is generated by the Hamiltonian vector fields $X_{h_{k}}, k \in$ $\{1, \ldots, m\}$. Using Theorem 2.9 and the fact that the torus $\mathbb{T}^{m}$ is abelian, we have that

$$
X_{\omega\left(X_{h_{k}}, X_{h_{l}}\right)}=-\left[X_{h_{k}}, X_{h_{l}}\right] \equiv 0 \Rightarrow \omega\left(X_{h_{k}}, X_{h_{l}}\right) \equiv \text { const. }, \forall k, l \in\{1, \ldots, m\} .
$$

Since $\mathbb{T}^{m}$ is compact, there is for each $k \in\{1, \ldots, m\}$ and on each $\mathbb{T}^{m}$-orbit a point $p_{k}$ where the function $\left.h_{k}\right|_{\text {orbit }}$ attains its maximum. Then

$$
\omega\left(X_{h_{k}}, X_{h_{l}}\right)=\left(d h_{k}\right)_{p_{k}}\left(X_{h_{l}}\right)=0 .
$$

Hence, the above constant is actually zero and each $\mathbb{T}^{m}$-orbit is indeed isotropic. This fact will be used below, in the proof of Proposition 2.1\%. 
Example 2.14. Consider $\left(\mathbb{R}^{2 n}, \omega_{\text {st }}\right)$, where

$$
\omega_{\mathrm{st}}=d u \wedge d v:=\sum_{j=1}^{n} d u_{j} \wedge d v_{j}
$$

as in Example 2.2, and its usual identification with $\mathbb{C}^{n}$ given by

$$
z_{j}=u_{j}+i v_{j}, j=1, \ldots, n .
$$

The standard $\mathbb{T}^{n}$-action $\tau_{\text {st }}$ on $\mathbb{R}^{2 n}$, given by

$$
\left(y_{1}, \ldots, y_{n}\right) \cdot\left(z_{1}, \ldots, z_{n}\right)=\left(e^{-i y_{1}} z_{1}, \ldots, e^{-i y_{n}} z_{n}\right),
$$

is Hamiltonian, with moment map $\mu_{\text {st }}: \mathbb{R}^{2 n} \rightarrow \mathbb{R}^{n}$ given by

$$
\mu_{\text {st }}\left(u_{1}, \ldots, u_{n}, v_{1}, \ldots, v_{n}\right)=\frac{1}{2}\left(u_{1}^{2}+v_{1}^{2}, \ldots, u_{n}^{2}+v_{n}^{2}\right) .
$$

Example 2.15. Consider projective space $\left(\mathbb{P}^{n}, \omega_{\mathrm{FS}}\right)$, with homogeneous coordinates $\left[z_{0} ; z_{1} ; \ldots ; z_{n}\right]$.

The $\mathbb{T}^{n}$-action $\tau_{\mathrm{FS}}$ on $\mathbb{P}^{n}$ given by

$$
\left(y_{1}, \ldots, y_{n}\right) \cdot\left[z_{0} ; z_{1} ; \ldots ; z_{n}\right]=\left[z_{0} ; e^{-i y_{1}} z_{1} ; \ldots ; e^{-i y_{n}} z_{n}\right]
$$

is Hamiltonian, with moment map $\mu_{\mathrm{FS}}: \mathbb{P}^{n} \rightarrow \mathbb{R}^{n}$ given by

$$
\mu_{\mathrm{FS}}\left[z_{0} ; z_{1} ; \ldots ; z_{n}\right]=\frac{1}{\|z\|^{2}}\left(\left\|z_{1}\right\|^{2}, \ldots,\left\|z_{n}\right\|^{2}\right) .
$$

Note that the image of $\mu_{\mathrm{FS}}$ is the convex hull of the images of the $n+1$ fixed points of the action, i.e. the standard simplex in $\mathbb{R}^{n}$.

Atiyah [5] and Guillemin-Sternberg [16] proved in 1982 the following Convexity Theorem.

Theorem 2.16. Let $(B, \omega)$ be a compact, connected, symplectic manifold, equipped with a Hamiltonian $\mathbb{T}^{m}$-action with moment map $\mu: B \rightarrow \mathbb{R}^{m}$. Then

(i) the level sets $\mu^{-1}(\lambda)$ of the moment map are connected (for any $\lambda \in \mathbb{R}^{m}$ );

(ii) the image $\mu(B) \subset \mathbb{R}^{m}$ of the moment map is the convex hull of the images of the fixed points of the action.

Toric Symplectic Manifolds. The following proposition motivates the definition of a toric symplectic manifold.

Proposition 2.17. If a symplectic manifold $(B, \omega)$ has an effective Hamiltonian $\mathbb{T}^{m}$-action, then $m \leq(\operatorname{dim} B) / 2$.

Proof.

Effective action $\Rightarrow$ there exist $m$-dimensional orbits.

Hamiltonian $\mathbb{T}^{m}$-action $\Rightarrow$ orbits are isotropic (see Remark 2.13).

Linear Algebra $\Rightarrow \operatorname{dim}($ isotropic orbit $) \leq \frac{1}{2} \operatorname{dim} B$.

Definition 2.18. A toric symplectic manifold is a connected symplectic manifold $\left(B^{2 n}, \omega\right)$, equipped with an effective Hamiltonian action of the $n$-torus,

$$
\tau: \mathbb{T}^{n} \cong \mathbb{R}^{n} / 2 \pi \mathbb{Z}^{n} \hookrightarrow \operatorname{Ham}(B, \omega),
$$

such that the corresponding moment map

$$
\mu: B \rightarrow \mathbb{R}^{n},
$$

well-defined up to a constant, is proper onto its convex image $P=\mu(B) \subset \mathbb{R}^{n}$.

Remark 2.19. The requirement that the moment map be "proper onto its convex image", something that is automatic for compact manifolds, makes the theory of non-compact toric symplectic manifolds analogous to the compact one (see [17]).

Example 2.20. $\left(\mathbb{R}^{2 n}, \omega_{\text {st }}\right)$, equipped with the standard Hamiltonian $\mathbb{T}^{n}$-action described in Example 2.14, is a non-compact toric symplectic manifold. 
Example 2.21. ( $\left.\mathbb{P}^{n}, \omega_{\mathrm{FS}}\right)$, equipped with the Hamiltonian $\mathbb{T}^{n}$-action described in Example 2.15, is a compact toric symplectic manifold.

Classification Theorem and Action-Angle Coordinates. Any toric symplectic manifold has an associated convex set, the image of the moment map of the torus action. The convex sets that arise in this way are characterized in the following definition.

Definition 2.22. A convex polyhedral set $P$ in $\mathbb{R}^{n}$ is called simple and integral if:

(1) there are $n$ edges meeting at each vertex $p$;

(2) the edges meeting at the vertex $p$ are rational, i.e. each edge is of the form $p+t_{i}, 0 \leq$ $t \leq \infty$, where $v_{i} \in \mathbb{Z}^{n}$

(3) the $v_{1}, \ldots, v_{n}$ in (2) can be chosen to be a $\mathbb{Z}$-basis of the lattice $\mathbb{Z}^{n}$.

$A$ facet is a face of $P$ of codimension one.

$A$ Delzant set is a simple and integral convex polyhedral set $P \subset \mathbb{R}^{n}$. A Delzant polytope is a compact Delzant set.

Two Delzant sets are isomorphic if one can be mapped to the other by a translation.

In 1988 Delzant [10] showed that any Delzant polytope determines a unique compact toric symplectic manifold. More precisely, if two compact toric symplectic manifolds have the same Delzant polytope, then there exists an equivariant symplectomorphism between them. This result can be generalized to the possibly non-compact setting of Definition 2.18 (see [17]).

Theorem 2.23. Let $(B, \omega, \tau)$ be a toric symplectic manifold, with moment map $\mu: B \rightarrow \mathbb{R}^{n}$. Then $P \equiv \mu(B)$ is a Delzant set.

Two toric symplectic manifolds are equivariant symplectomorphic (with respect to a fixed torus acting on both) if and only if their associated Delzant sets are isomorphic. Moreover, every Delzant set arises from some toric symplectic manifold.

Remark 2.24. One can use the work of Lerman and Tolman [19] to generalize Theorem 2.23 to orbifolds. The outcome is a classification of symplectic toric orbifolds via labeled Delzant sets, i.e. convex polyhedral sets, as in Definition 2.22, with "ZZ-basis" in (3) replaced by "Q্Q-basis" and with a positive integer label attached to each facet.

Each facet $F$ of a labeled Delzant set $P \subset \mathbb{R}^{n}$ determines a unique lattice vector $\nu_{F} \in \mathbb{Z}^{n}$ : the primitive inward pointing normal lattice vector. A convenient way of thinking about a positive integer label $m_{F} \in \mathbb{N}$ associated to $F$ is by dropping the primitive requirement from this lattice vector: consider $m_{F} \nu_{F}$ instead of $\nu_{F}$.

In other words, a labeled Delzant set can be defined as a rational simple polyhedral set $P \subset \mathbb{R}^{n}$ with an inward pointing normal lattice vector associated to each of its facets.

The proof gives an explicit symplectic reduction construction of a canonical model for each toric symplectic manifold, i.e. it associates to each Delzant set $P$ an explicit toric symplectic manifold $\left(B_{P}, \omega_{P}, \tau_{P}\right)$ with moment map $\mu_{P}: B_{P} \rightarrow P$. One can use these canonical models to derive general properties of toric symplectic manifolds. For example, let $\breve{P}$ denote the interior of $P$, and consider $\breve{B}_{P} \subset B_{P}$ defined by $\breve{B}_{P}=\mu_{P}^{-1}(\breve{P})$. One easily checks that $\breve{B}_{P}$ is an open dense subset of $B_{P}$, consisting of all the points where the $\mathbb{T}^{n}$-action is free. It can be described as

$$
\breve{B}_{P} \cong \breve{P} \times \mathbb{T}^{n}=\left\{(x, y): x \in \breve{P} \subset \mathbb{R}^{n}, y \in \mathbb{R}^{n} / 2 \pi \mathbb{Z}^{n}\right\}
$$

where $(x, y)$ are symplectic action-angle coordinates for $\omega_{P}$, i.e.

$$
\left.\omega_{P}\right|_{\breve{B}}=d x \wedge d y=\sum_{j=1}^{n} d x_{j} \wedge d y_{j} .
$$

Hence, one has a global equivariant Darboux's Theorem in this toric context. Note that in these action-angle coordinates the moment map is simply given by

$$
\mu_{P}(x, y)=x,
$$

i.e. projection in the action coordinates. 


\section{Toric Kähler Metrics}

\section{Linear Compatible Complex Structures.}

Definition 3.1. A compatible complex structure on a symplectic vector space $(V, \omega)$ is a complex structure $J$ on $V$, i.e. $J \in \operatorname{End}(V))$ with $J^{2}=-\mathrm{Id}$, such that

$$
\langle\cdot, \cdot\rangle_{J}:=\omega(\cdot, J \cdot)
$$

is an inner product on $V$. This is equivalent to

$$
\omega(J \cdot, J \cdot)=\omega(\cdot, \cdot) \quad \text { and } \quad \omega(v, J v)>0, \forall 0 \neq v \in V .
$$

The set of all compatible complex structures on a symplectic vector space $(V, \omega)$ will be denoted by $\mathcal{J}(V, \omega)$.

The symplectic linear group $S p(V, \omega)$ acts on $\mathcal{J}(V, \omega)$ by conjugation:

$$
\begin{aligned}
S p(V, \omega) \times \mathcal{J}(V, \omega) & \rightarrow \mathcal{J}(V, \omega) \\
(\Phi, J) & \mapsto \Phi J \Phi^{-1}
\end{aligned}
$$

This action can be easily seen to be transitive and, if we fix $J_{0} \in \mathcal{J}(V, \omega)$ and corresponding inner product $\langle\cdot, \cdot\rangle_{0}$, we have that

$$
\mathcal{J}(V, \omega)=S p(V, \omega) / U\left(V, \omega,\langle\cdot, \cdot\rangle_{0}\right)
$$

where $U\left(V, \omega,\langle\cdot, \cdot\rangle_{0}\right)=S p(V, \omega) \cap O\left(V,\langle\cdot, \cdot\rangle_{0}\right)$ is the unitary group. $\mathcal{J}(V, \omega)$ is a symmetric space and admits a beautiful explicit description due to C.L. Siegel [23].

Definition 3.2. The Siegel upper half space $\mathcal{S}_{n}$ is the open contractible subset of the complex vector space of complex symmetric matrices defined by

$$
\begin{aligned}
\mathcal{S}_{n}:=\{Z=R+i S: & R \text { and } S \text { are real symmetric }(n \times n) \text { matrices, } \\
& \text { with } S \text { positive definite }\} .
\end{aligned}
$$

Choose a symplectic basis for $(V, \omega)$, i.e. an isomorphism $(V, \omega) \cong\left(\mathbb{R}^{2 n}, \omega_{\text {st }}\right)$, and identify the symplectic linear group $S p(V, \omega)$ with the matrix group $S p(2 n, \mathbb{R})$ consisting of $(2 n \times 2 n)$ real matrices $\Phi$ such that

where

$$
\Phi^{t} \cdot \omega_{0} \cdot \Phi=\omega_{0}
$$

$$
\omega_{0}=\left[\begin{array}{ccc}
0 & \vdots & \text { Id } \\
\ldots \ldots & \ldots & \ldots \\
-\mathrm{Id} & \vdots & 0
\end{array}\right]
$$

is the matrix form of $\omega_{\text {st }}$ written in $(n \times n)$ blocks. We will also write any $\Phi \in S p(2 n, \mathbb{R})$ in $(n \times n)$ blocks:

$$
\Phi=\left[\begin{array}{ccc}
A & \vdots & B \\
\cdots & \ldots & \ldots \\
C & \vdots & D
\end{array}\right] .
$$

The following proposition is proved in [23].

Proposition 3.3. $S p(2 n, \mathbb{R})$ acts on $\mathcal{S}_{n}$ by linear fractional transformations:

$$
\begin{aligned}
S p(2 n, \mathbb{R}) \times \mathcal{S}_{n} & \rightarrow \mathcal{S}_{n} \\
(\Phi, Z) & \mapsto \Phi(Z):=(A Z+B) \cdot(C Z+D)^{-1} .
\end{aligned}
$$

This action is transitive and the isotropy group of $i \operatorname{Id} \in \mathcal{S}_{n}$ is $U(n) \subset S p(2 n, \mathbb{R})$ :

$$
U(n)=S p(2 n, \mathbb{R}) \cap O(n)=\left\{\Phi \in S p(2 n, \mathbb{R}): \Phi^{t} \cdot \Phi=\operatorname{Id}\right\} .
$$

Hence,

$$
\mathcal{S}_{n} \cong S p(2 n, \mathbb{R}) / U(n)
$$


Given $Z=R+i S \in \mathcal{S}_{n}$, define

$$
\Phi_{Z}:=\left[\begin{array}{ccc}
S^{1 / 2} & \vdots & R S^{-1 / 2} \\
\cdots & \ldots & \ldots \\
0 & \vdots & S^{-1 / 2}
\end{array}\right] \in S p(2 n, \mathbb{R}) .
$$

Under the action of $S p(2 n, \mathbb{R})$ on $\mathcal{S}_{n}$, we have that

$$
\Phi_{Z}(i \mathrm{Id})=Z \text {. }
$$

Let $J_{0} \in \mathcal{J}\left(\mathbb{R}^{2 n}, \omega_{\text {st }}\right)$ be given by

$$
J_{0}=\left[\begin{array}{ccc}
0 & \vdots & -\operatorname{Id} \\
\cdots & \ldots & \ldots \\
\operatorname{Id} & \vdots & 0
\end{array}\right]
$$

For each $Z \in \mathcal{S}_{n}$, define $J_{Z} \in \mathcal{J}\left(\mathbb{R}^{2 n}, \omega_{\mathrm{st}}\right)$ by

$$
J_{Z}:=\left(J_{0} \cdot \Phi_{Z}\right) \cdot J_{0} \cdot\left(J_{0} \cdot \Phi_{Z}\right)^{-1}=\left[\begin{array}{ccc}
-S^{-1} R & \vdots & -S^{-1} \\
\ldots \cdots & \ldots & \ldots \\
R S^{-1} R+S & \vdots & R S^{-1}
\end{array}\right] .
$$

This defines a bijection

$$
\begin{aligned}
\mathcal{S}_{n} & \rightarrow \mathcal{J}\left(\mathbb{R}^{2 n}, \omega_{\text {st }}\right) \\
Z & \mapsto J_{Z}
\end{aligned}
$$

which, up to $J_{0}$-conjugation, is equivariant with respect to the $S p(2 n, \mathbb{R})$-action on both spaces. More precisely, if $\Phi \in S p(2 n, \mathbb{R})$ then

$$
\Phi \cdot J_{Z} \cdot \Phi^{-1}=J_{Z^{\prime}} \Leftrightarrow Z^{\prime}=\left(J_{0}^{-1} \cdot \Phi \cdot J_{0}\right)(Z) .
$$

In particular, $\mathcal{J}\left(\mathbb{R}^{2 n}, \omega_{\mathrm{st}}\right)$ is a contractible space and, for any symmetric $(n \times n)$ real matrix $U$, we have that

$$
\Phi=\left[\begin{array}{ccc}
I & \vdots & 0 \\
\cdots & \cdots & . \\
U & \vdots & I
\end{array}\right] \in S p(2 n, \mathbb{R}) \text { and } \Phi \cdot J_{Z} \cdot \Phi^{-1}=J_{(Z-U)} .
$$

This will be relevant below.

\section{Toric Compatible Complex Structures.}

Definition 3.4. A compatible almost complex structure on a symplectic manifold $(B, \omega)$ is an almost complex structure $J$ on $B$, i.e. $J \in \Gamma(\operatorname{End}(T B))$ with $J^{2}=-\mathrm{Id}$, such that

$$
\langle\cdot, \cdot\rangle_{J}:=\omega(\cdot, J \cdot)
$$

is a Riemannian metric on $B$. This is equivalent to $\omega(J \cdot, J \cdot)=\omega(\cdot, \cdot)$ and $\omega(X, J X)>0, \forall 0 \neq$ $X \in T B$.

The space of all compatible almost complex structures on a symplectic manifold $(B, \omega)$ will be denoted by $\mathcal{J}(B, \omega)$.

\section{Remark 3.5.}

(i) The fact that $\mathcal{J}\left(\mathbb{R}^{2 n}, \omega_{\text {st }}\right)$ is contractible implies that $\mathcal{J}(B, \omega)$ is non-empty, infinitedimensional and contractible, for any symplectic manifold $(B, \omega)$.

(ii) A Kähler manifold is a symplectic manifold $(B, \omega)$ with an integrable compatible complex structure $J$, i.e. one that is locally isomorphic to the standard complex structure $J_{0}$ on $\mathbb{R}^{2 n}$. Note that (1) gives the standard isomorphism $\left(\mathbb{R}^{2 n}, J_{0}\right) \cong \mathbb{C}^{n}$.

(iii) The space of integrable compatible complex structures on a symplectic manifold $(B, \omega)$ will be denoted by $\mathcal{I}(B, \omega) \subset \mathcal{J}(B, \omega)$. 
(iv) In general, $\mathcal{I}(B, \omega)$ can be empty.

Definition 3.6. A toric compatible complex structure on a toric symplectic manifold $\left(B^{2 n}, \omega, \tau\right)$ is a $\mathbb{T}^{n}$-invariant $J \in \mathcal{I}(B, \omega) \subset \mathcal{J}(B, \omega)$. The space of all such will be denoted by $\mathcal{I}^{\mathbb{T}^{n}}(B, \omega) \subset$ $\mathcal{J}^{\mathbb{T}^{n}}(B, \omega)$.

Remark 3.7. It follows from the classification in Theorem 2.23, more precisely from the explicit symplectic reduction construction of the canonical model for any compact toric symplectic manifold $\left(B^{2 n}, \omega, \tau\right)$, that $\mathcal{I}^{\mathbb{T}^{n}}(B, \omega)$ is always non-empty.

Local Form of Toric Compatible Complex Structures. It follows from the above bijection between $\mathcal{J}\left(\mathbb{R}^{2 n}, \omega_{\text {st }}\right)$ and the Siegel upper half space $\mathcal{S}_{n}$ that any $J \in \mathcal{J}^{\mathbb{T}^{n}}\left(\breve{B},\left.\omega\right|_{\breve{B}}\right)$ can be written in action-angle coordinates $(x, y)$ on $\breve{B} \cong \breve{P} \times \mathbb{T}^{n}$ as

$$
J=\left[\begin{array}{ccc}
-S^{-1} R & \vdots & -S^{-1} \\
\ldots \ldots \ldots \ldots \ldots \ldots \\
R S^{-1} R+S & \vdots & R S^{-1}
\end{array}\right]
$$

where $R=R(x)$ and $S=S(x)$ are real symmetric $(n \times n)$ matrices, with $S$ positive definite.

For integrable toric compatible complex structures we have that:

$$
\begin{gathered}
J \in \mathcal{I}^{\mathbb{T}^{n}} \subset \mathcal{J}^{\mathbb{T}^{n}} \Leftrightarrow \frac{\partial Z_{i j}}{\partial x_{k}}=\frac{\partial Z_{i k}}{\partial x_{j}} \\
\Leftrightarrow \exists f: \breve{P} \rightarrow \mathbb{C}, f(x)=r(x)+i s(x) \text {, such that } \\
Z_{i j}=\frac{\partial^{2} f}{\partial x_{i} \partial x_{j}}=\frac{\partial^{2} r}{\partial x_{i} \partial x_{j}}+i \frac{\partial^{2} s}{\partial x_{i} \partial x_{j}}=R_{i j}+i S_{i j} .
\end{gathered}
$$

Any real function $h: \breve{P} \rightarrow \mathbb{R}$ is the Hamiltonian of a 1-parameter family

$$
\phi_{t}: \breve{B} \rightarrow \breve{B}
$$

of $\mathbb{T}^{n}$-equivariant symplectomorphisms. These are given in action-angle coordinates $(x, y)$ on $\breve{B} \cong \breve{P} \times \mathbb{T}^{n}$ by

$$
\phi_{t}(x, y)=\left(x, y-t \frac{\partial h}{\partial x}\right) .
$$

Hence, it follows from (3) that the natural action of $\phi_{t}$ on $\mathcal{J}^{\mathbb{T}^{n}}$, given by

$$
\phi_{t} \cdot J=\left(d \phi_{t}\right) \circ J \circ\left(d \phi_{t}\right)^{-1},
$$

corresponds in the Siegel upper half space parametrization to

$$
\phi_{t} \cdot(Z=R+i S)=(R+t H)+i S,
$$

where

$$
H=\left(h_{i j}\right)=\left(\frac{\partial^{2} h}{\partial x_{i} \partial x_{j}}\right) .
$$

This implies that, for any integrable $J \in \mathcal{I}^{\mathbb{T}^{n}}$, there exist action-angle coordinates $(x, y)$ on $\breve{B} \cong \breve{P} \times \mathbb{T}^{n}$ such that $R \equiv 0$, i.e. such that

$$
J=\left[\begin{array}{ccc}
0 & \vdots & -S^{-1} \\
\ldots & \ldots & \ldots \\
S & \vdots & 0
\end{array}\right]
$$

with

for some

$$
S=S(x)=\left(s_{i j}(x)\right)=\left(\frac{\partial^{2} s}{\partial x_{i} \partial x_{j}}\right)
$$

real potential function $\quad s: \breve{P} \rightarrow \mathbb{R}$. 
Holomorphic coordinates for $J$ are given in this case by

$$
z(x, y)=u(x, y)+i v(x, y)=\frac{\partial s}{\partial x}(x)+i y .
$$

The corresponding Riemannian (Kähler) metric

$$
\langle\cdot, \cdot\rangle_{J}:=\omega(\cdot, J \cdot)
$$

on $\breve{B} \cong \breve{P} \times \mathbb{T}^{n}$ can the be written in matrix form as

$$
\omega_{0} \cdot J=\left[\begin{array}{ccc}
0 & \vdots & \operatorname{Id} \\
\ldots & \ldots & \ldots \\
-\mathrm{Id} & \vdots & 0
\end{array}\right] \cdot\left[\begin{array}{ccc}
0 & \vdots & -S^{-1} \\
\ldots & \ldots & \ldots \\
S & \vdots & 0
\end{array}\right]=\left[\begin{array}{ccc}
S & \vdots & 0 \\
\ldots \ldots & \ldots \\
0 & \vdots & S^{-1}
\end{array}\right]
$$

with

$$
S=\left(\frac{\partial^{2} s}{\partial x_{i} \partial x_{j}}\right)
$$

Definition 3.8. We will call such a potential function

$$
s: \breve{P} \rightarrow \mathbb{R}
$$

the symplectic potential of both the complex structure $J$ and the metric $\langle\cdot, \cdot\rangle_{J}$

Remark 3.9. This particular way to arrive at the above local form for any $J \in \mathcal{I}^{\mathbb{T}^{n}}$ is due to Donaldson [12], and illustrates a small part of his formal general framework for the action of the symplectomorphism group of a symplectic manifold on its space of compatible complex structures (cf. [11]).

Example 3.10. Consider the standard linear complex structure $J_{0} \in \mathcal{I}^{\mathbb{T}^{n}}\left(\mathbb{R}^{2 n}, \omega_{\mathrm{st}}\right)$ given by (2). In action-angle coordinates $(x, y)$ on

$$
\breve{\mathbb{R}}^{2 n}=\left(\mathbb{R}^{2} \backslash\{(0,0)\}\right)^{n} \cong\left(\mathbb{R}^{+}\right)^{n} \times \mathbb{T}^{n}=\breve{P} \times \mathbb{T}^{n},
$$

its symplectic potential is given by

$$
\begin{aligned}
s: \breve{P}=\left(\mathbb{R}^{+}\right)^{n} \longrightarrow \mathbb{R} \\
x=\left(x_{1}, \ldots, x_{n}\right) \longmapsto s(x)=\frac{1}{2} \sum_{i=1}^{n} x_{i} \log \left(x_{i}\right) .
\end{aligned}
$$

Hence, in these action-angle coordinates, the standard complex structure has the matrix form

$$
J_{0}=\left[\begin{array}{ccc}
0 & \vdots & \operatorname{diag}\left(-2 x_{i}\right) \\
\ldots \ldots \ldots \ldots \ldots \ldots \ldots \ldots & \ldots \ldots \ldots
\end{array}\right]
$$

while the standard flat Euclidean metric becomes

$$
\left[\begin{array}{ccc}
\operatorname{diag}\left(1 / 2 x_{i}\right) & \vdots & 0 \\
\ldots \ldots \ldots \ldots \ldots \ldots \ldots \ldots & \ldots \ldots
\end{array}\right]
$$

Symplectic Potentials for Compact Toric Symplectic Manifolds. The proof of Theorem 2.23 associates to each Delzant set $P \subset \mathbb{R}^{n}$, via an explicit symplectic reduction construction, a canonical Kähler toric manifold

$$
\left(B_{P}^{2 n}, \omega_{P}, \tau_{P}, \mu_{P}, J_{P}\right) \text { such that } \mu_{P}\left(B_{P}\right)=P .
$$

In [15] Guillemin gave an explicit formula for the symplectic potential of this canonical Kähler metric. To write it down one just needs some simple combinatorial data that can be easily obtained directly from the polytope $P$. 
Let $F_{i}$ denote the $i$-th facet (codimension-1 face) of the polytope $P$. The affine defining function of $F_{i}$ is the function

$$
\begin{aligned}
\ell_{i}: \mathbb{R}^{n} & \longrightarrow \mathbb{R} \\
x & \longmapsto \ell_{i}(x)=\left\langle x, \nu_{i}\right\rangle-\lambda_{i},
\end{aligned}
$$

where $\nu_{i} \in \mathbb{Z}^{n}$ is a primitive inward pointing normal to $F_{i}$ and $\lambda_{i} \in \mathbb{R}$ is such that $\left.\ell_{i}\right|_{F_{i}} \equiv 0$. Note that $\left.\ell_{i}\right|_{\breve{P}}>0$.

Theorem 3.11. In appropriate action-angle coordinates $(x, y)$, the canonical symplectic potential $s_{P}: \breve{P} \rightarrow \mathbb{R}$ for $\left.J_{P}\right|_{\breve{P}}$ is given by

$$
s_{P}(x)=\frac{1}{2} \sum_{i=1}^{d} \ell_{i}(x) \log \ell_{i}(x),
$$

where $d$ is the number of facets of $P$.

Example 3.12. The symplectic potential presented in Example 3.10 for the standard flat Euclidean metric on $\mathbb{R}^{2 n}$ is the canonical symplectic potential of the corresponding Delzant set $P=\left(\mathbb{R}_{0}^{+}\right)^{n} \subset \mathbb{R}^{n}$.

Example 3.13. For projective space $\mathbb{P}^{n}$ the polytope $P \subset \mathbb{R}^{n}$ can be taken to be the standard simplex, with defining affine functions

$$
\ell_{i}(x)=x_{i}, i=1, \ldots, n, \quad \text { and } \quad \ell_{n+1}(x)=1-r,
$$

where $r=\sum_{i} x_{i}$.

The canonical symplectic potential $s_{P}: \breve{P} \rightarrow \mathbb{R}$, given by

$$
s_{P}(x)=\frac{1}{2} \sum_{i=1}^{n} x_{i} \log x_{i}+\frac{1}{2}(1-r) \log (1-r),
$$

defines the standard complex structure $J_{F S}$ and Fubini-Study metric on $\mathbb{P}^{n}$.

Theorem 3.15 below provides the symplectic version of the $\partial \bar{\partial}$-lemma in this toric context, characterizing the symplectic potentials that correspond to toric compatible complex structures on a toric symplectic manifold. It is an immediate extension to our possibly non-compact setting of the compact version proved in [2]. To properly state it we need the following definition.

Definition 3.14. Let $(B, \omega, \tau)$ be a symplectic toric manifold and denote by

$$
Y_{1}, \ldots, Y_{n} \in \mathcal{X}_{H}(B, \omega)
$$

the Hamiltonian vector fields generating the torus action. A toric compatible complex structure $J \in \mathcal{I}^{\mathcal{T}}(B, \omega)$ is said to be complete if the $J$-holomorphic vector fields

$$
J Y_{1}, \ldots, J Y_{n} \in \mathcal{X}(B)
$$

are complete. The space of all complete toric compatible complex structures on $(B, \omega, \tau)$ will be denoted by $\mathcal{I}_{c}^{\mathcal{T}}(B, \omega)$.

Theorem 3.15. Let $J$ be any complete compatible toric complex structure on the symplectic toric manifold $\left(B_{P}, \omega_{P}, \tau_{P}\right)$. Then, in suitable action-angle $(x, y)$-coordinates on $\breve{B}_{P} \cong \breve{P} \times \mathbb{T}^{n}, J$ is given by a symplectic potential $s \in C^{\infty}(\breve{P})$ of the form

$$
s(x)=s_{P}(x)+h(x),
$$

where $s_{P}$ is given by Theorem 3.11, $h$ is smooth on the whole $P$, and the matrix $S=\operatorname{Hess}_{x}(s)$ is positive definite on $\breve{P}$ and has determinant of the form

$$
\operatorname{Det}(S)=\left(\delta \prod_{r=1}^{d} \ell_{r}\right)^{-1},
$$

with $\delta$ being a smooth and strictly positive function on the whole $P$. 
Conversely, any such potential s determines a (not necessarily complete) complex structure on $\breve{B}_{P} \cong \breve{P} \times \mathbb{T}^{n}$, that extends uniquely to a well-defined compatible toric complex structure $J$ on the toric symplectic manifold $\left(B_{P}, \omega_{P}, \tau_{P}\right)$.

Remark 3.16. If one takes into account Remark 2.24, the word "manifold" can be replaced by "orbifold" in Theorems 3.11 and 3.15 (see [3]).

Remark 3.17. There is no immediate relation between completeness of a toric compatible complex structure and completeness of the associated toric Kähler metric. See Remark 4.1.

Scalar Curvature. We now recall from [1] a particular formula for the scalar curvature in actionangle $(x, y)$-coordinates. A Kähler metric of the form (5) has scalar curvature $S c$ given by ${ }^{1}$

$$
S c=-\sum_{j, k} \frac{\partial}{\partial x_{j}}\left(g^{j k} \frac{\partial \log \operatorname{Det}(S)}{\partial x_{k}}\right),
$$

which after some algebraic manipulations becomes the more compact

$$
S c=-\sum_{j, k} \frac{\partial^{2} s^{j k}}{\partial x_{j} \partial x_{k}}
$$

where the $s^{j k}, 1 \leq j, k \leq n$, are the entries of the inverse of the matrix $S=\operatorname{Hess}_{x}(s), s \equiv$ symplectic potential. See [11] for an appropriate interpretation of this formula for the scalar curvature.

Symplectic Potentials and Affine Transformations. Because the Delzant set $P \subset \mathbb{R}^{n}$ of a symplectic toric manifold is only really well defined up to translations (i.e. additions of constants to the moment map) and $S L(n, \mathbb{Z})$ transformations (i.e. changes of basis of the torus $\mathbb{T}^{n}=\mathbb{R}^{n} / 2 \pi \mathbb{Z}^{n}$ ), symplectic potentials should transform naturally under these type of maps. While the effect of translations is trivial to analyse, the effect of $S L(n, \mathbb{Z})$ transformations is more interesting. In fact:

symplectic potentials transform quite naturally under any $G L(n, \mathbb{R})$ linear transformation.

Let $T \in G L(n, \mathbb{R})$ and consider the linear symplectic change of action-angle coordinates

$$
x:=T^{-1} x^{\prime} \text { and } y:=T^{t} y^{\prime} .
$$

Then

becomes

$$
P^{\prime}=\bigcap_{a=1}^{d}\left\{x^{\prime} \in \mathbb{R}^{n}: \ell_{a}^{\prime}\left(x^{\prime}\right):=\left\langle x^{\prime}, \nu_{a}^{\prime}\right\rangle+\lambda_{a}^{\prime} \geq 0\right\}
$$

$$
P:=T^{-1}\left(P^{\prime}\right)=\bigcap_{a=1}^{d}\left\{x \in \mathbb{R}^{n}: \ell_{a}(x):=\left\langle x, \nu_{a}\right\rangle+\lambda_{a} \geq 0\right\}
$$

with

$$
\nu_{a}=T^{t} \nu_{a}^{\prime} \text { and } \quad \lambda_{a}=\lambda_{a}^{\prime},
$$

and symplectic potentials transform by

$$
s=s^{\prime} \circ T \quad\left(\text { in particular, } s_{P}=s_{P}^{\prime} \circ T\right) .
$$

The corresponding Hessians are related by

$$
S=T^{t}\left(S^{\prime} \circ T\right) T
$$

and

$$
S c=S c^{\prime} \circ T \text {. }
$$

\footnotetext{
${ }^{1}$ The normalization for the value of the scalar curvature we are using here differs from the one used in [1, 2] by a factor of $1 / 2$.
} 


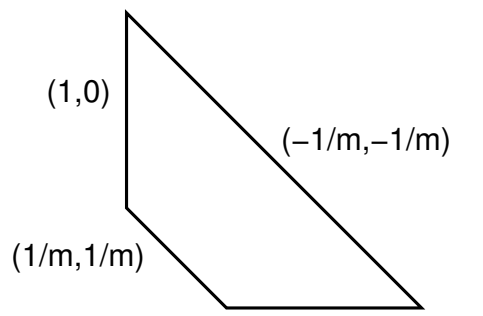

$(0,1)$

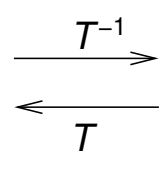

$(1,0)$

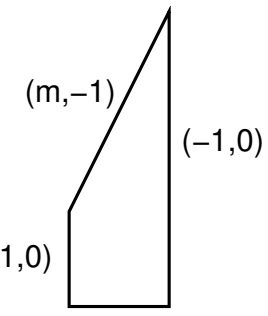

$(0,1)$

FiguRE 1. Hirzebruch surfaces.

Example 3.18. Figure 1 illustrates two equivalent descriptions of a toric symplectic rational ruled 4-manifold or, equivalently, of a Hirzebruch surface

$$
H_{m}^{2}:=\mathbb{P}(\mathcal{O}(-m) \oplus \mathbb{C}) \rightarrow \mathbb{P}^{1}, m \in \mathbb{N} .
$$

The linear map $T \in G L(2, \mathbb{R})$ relating the two is given by

$$
T=\left[\begin{array}{cc}
m & -1 \\
0 & 1
\end{array}\right]
$$

The inward pointing normal that should be considered for each facet is specified. The polytope on the right is a standard Delzant polytope for the Hirzebruch surface $H_{m}^{2}$. The polytope on the left is very useful for the Kähler metric constructions of section 5 and was implicitly used by Calabi in $[7]$.

\section{Toric Constant Curvature Metrics in Real Dimension 2}

In real dimension 2 any orientable Riemannian manifold is Kähler, since its area form is a symplectic form and oriented rotation by $\pi / 2$ on each tangent plane is a compatible complex structure. In this section we write down the symplectic potentials that give rise to toric constant (scalar) curvature metrics in real dimension 2 and identify the underlying toric symplectic manifolds $B$. This will be a warm-up for the higher dimensional examples presented in section 5 .

According to formula (6) for the scalar curvature, we are looking for symplectic potentials $s: \breve{P} \subset \mathbb{R} \rightarrow \mathbb{R}$ such that $s^{\prime \prime}>0$ and

$$
-\left(\frac{1}{s^{\prime \prime}(x)}\right)^{\prime \prime}=2 k,
$$

where $k \in \mathbb{R}$ denotes the Gauss curvature. This implies that

$$
s^{\prime \prime}(x)=-\frac{1}{k x^{2}-2 b x-c}, b, c \in \mathbb{R}
$$

where $x \in \breve{P} \subset \mathbb{R}$ is such that $s^{\prime \prime}(x)>0$.

Cylinders. Suppose that $k=b=0$. Then

$$
s^{\prime \prime}(x)=\frac{1}{c}>0 \Rightarrow c>0 \quad \text { and } \quad x \in \mathbb{R} .
$$

This means that $P=\mathbb{R}$ and $s: \breve{P}=\mathbb{R} \rightarrow \mathbb{R}$ can be written as

Hence

$$
s(x)=\frac{x^{2}}{2 c} .
$$

and the metric is given in matrix form by

i.e. we get a flat cylinder of radius $\sqrt{c}$.

$$
\left[\begin{array}{cc}
1 / c & 0 \\
0 & c
\end{array}\right]
$$


Cones. Suppose that $k=0$ and $b \neq 0$. Then, modulo a translation and possible sign change in the action variable $x$, we can assume that $c=0, b>0$ and

$$
s^{\prime \prime}(x)=\frac{1}{2 b x}>0 \Rightarrow x>0 .
$$

This means that $P=[0,+\infty[$ and $s: \breve{P}=] 0,+\infty[\rightarrow \mathbb{R}$ can be written as

$$
s(x)=\frac{1}{b} \cdot \frac{1}{2} x \log x .
$$

If $b=1$ this is the canonical symplectic potential giving the flat Euclidean metric on $\mathbb{R}^{2}$ (cf. Example 3.10). In general, as explained in [3], this is the symplectic potential of a cone metric of angle $\pi b$ on $\mathbb{R}^{2}$, given in matrix form by

$$
\left[\begin{array}{cc}
\frac{1}{2 b x} & 0 \\
0 & 2 b x
\end{array}\right]
$$

When $b=1 / p$ with $p \in \mathbb{N}$, this corresponds to an orbifold flat metric on $\mathbb{R}^{2} / \mathbb{Z}_{p}$ (see [3]).

Footballs. Suppose that $k>0$. Then, modulo a translation in the action variable $x$, we can assume that $b=0$ and

$$
s^{\prime \prime}(x)=\frac{1}{c-k x^{2}}>0 \Rightarrow c>0 \text { and }-\sqrt{c / k}<x<\sqrt{c / k} .
$$

This means that $P=[-\sqrt{c / k}, \sqrt{c / k}]$ and $s: \breve{P}=]-\sqrt{c / k}, \sqrt{c / k}[\rightarrow \mathbb{R}$ can be written as

$$
s(x)=\frac{1}{\sqrt{c k}} \cdot \frac{1}{2}[(x+\sqrt{c / k}) \log (x+\sqrt{c / k})+(-x+\sqrt{c / k}) \log (-x+\sqrt{c / k})] .
$$

If $c=1 / k$ this is the canonical symplectic potential giving the smooth round european football metric of total area $4 \pi / k$ and constant Gauss curvature $k$ on $\mathbb{P}^{1} \equiv \mathbb{S}^{2}$ (cf. Example 3.13). In general, this is the symplectic potential of a singular american football metric of angle $\pi \sqrt{c k}$ at the "poles".

Hyperbolic Metrics. Suppose that $k<0$. Then, modulo a translation in the action variable $x$, we can assume that $b=0$ and

$$
s^{\prime \prime}(x)=\frac{1}{c-k x^{2}} .
$$

If $c>0$ then $s^{\prime \prime}(x)>0, \forall x \in \mathbb{R}$, which means that $P=\mathbb{R}$ and $s: \breve{P}=\mathbb{R} \rightarrow \mathbb{R}$ can be written as

$$
s(x)=\sqrt{\frac{-1}{c k}} \arctan \left(\sqrt{\frac{-k}{c}} x\right) .
$$

This is the symplectic potential of a metric of constant Gauss curvature $k<0$ on

$$
B=\breve{B}=\breve{P} \times \mathbb{T}^{1}=\mathbb{R} \times S^{1}=\{(x, y): x \in \mathbb{R}, y \in \mathbb{R} / 2 \pi \mathbb{Z}\},
$$

i.e. an hyperboloid.

If $c<0$ then

$$
\left.s^{\prime \prime}(x)>0 \Rightarrow x \in\right]-\infty,-\sqrt{c / k}[\cup] \sqrt{c / k},+\infty[
$$

Hence, up to a sign change in the action variable $x$, we may assume that $P=[\sqrt{c / k},+\infty[$ and $s: \breve{P}=] \sqrt{c / k},+\infty[\rightarrow \mathbb{R}$ can be written as

$$
s(x)=\frac{1}{\sqrt{c k}} \cdot \frac{1}{2}[(x-\sqrt{c / k}) \log (x-\sqrt{c / k})-(x+\sqrt{c / k}) \log (x+\sqrt{c / k})] .
$$


If $c=1 / k$ this is the symplectic potential of the hyperbolic metric of constant Gauss curvature $k<0$ on $\mathbb{R}^{2}$. In other words, in the action-angle coordinates $(x, y)$ of this symplectic model, the hyperbolic metric is given in matrix form by

$$
\left[\begin{array}{cc}
\frac{-k}{(k x)^{2}-1} & 0 \\
0 & \frac{(k x)^{2}-1}{-k}
\end{array}\right] .
$$

More generally, i.e. when $c \neq 1 / k$, we get singular hyperbolic metrics on $\mathbb{R}^{2}$, with a cone singularity of angle $\pi \sqrt{c k}$ at the origin.

Remark 4.1. This case illustrates the fact that there is no immediate relation between completeness of a toric compatible complex structure and completeness of the associated toric Kähler metric. Here the metric is complete but the complex structure is not. In fact, it easily follows from (4) that $\mathbb{R}^{2}$ with this complex structure is biholomorphic to an open bounded disc $D \subset \mathbb{C}$.

If $c=0$ we have that

$$
s^{\prime \prime}(x)=\frac{1}{-k x^{2}}>0, \forall x \neq 0,
$$

and $s: \breve{P}=] 0,+\infty[\rightarrow \mathbb{R}$ can be written as

$$
s(x)=\frac{1}{k} \log (x) .
$$

This is the symplectic potential of a complete hyperbolic cusp metric on $\breve{B}=] 0,+\infty\left[\times S^{1}\right.$, given in matrix form by

$$
\left[\begin{array}{cc}
\frac{1}{-k x^{2}} & 0 \\
0 & -k x^{2}
\end{array}\right]
$$

\section{Calabi's Family of Extremal Kähler Metrics}

In [7], Calabi introduced the notion of extremal Kähler metrics. These are defined, for a fixed closed complex manifold $(M, J)$, as critical points of the square of the $L^{2}$-norm of the scalar curvature, considered as a functional on the space of all symplectic Kähler forms $\omega$ in a fixed Kähler class $\Omega \in H^{2}(M, \mathbb{R})$. The extremal Euler-Lagrange equation is equivalent to the gradient of the scalar curvature being an holomorphic vector field (see [6]), and so these metrics generalize constant scalar curvature Kähler metrics. Moreover, Calabi showed in [8] that extremal Kähler metrics are always invariant under a maximal compact subgroup of the group of holomorphic transformations of $(M, J)$. Hence, on a toric manifold, extremal Kähler metrics are automatically toric Kähler metrics, and one should be able to write them down using the previous action-angle coordinates framework.

In this section, following [1], we will do that for the 4-parameter family of $U(n)$-invariant extremal Kähler metrics constructed by Calabi in [7]. Calabi used this family to put extremal Kähler metrics on

$$
\mathbb{P}(\mathcal{O}(-m) \oplus \mathbb{C}) \longrightarrow \mathbb{P}^{n-1}, n, m \in \mathbb{N},
$$

for any Kähler class. In particular, when $n=2$, on all Hirzebruch surfaces (cf. Example 3.18). As we will see here, this family can be used to write down many other interesting extremal Kähler metrics, including the non-compact, cohomogeneity one, constant scalar curvature examples that were later constructed by LeBrun [18], Pedersen-Poon [21] and Simanca [24]. Using the actionangle coordinates set-up for toric Sasaki geometry developed in [20], one can show [4] that Calabi's family also contains a family of Kähler-Einstein metrics directly related to the Sasaki-Einstein metrics constructed in 2004 by Gauntlett-Martelli-Sparks-Waldram [13, 14].

Calabi's Family in Action-Angle Coordinates. Consider symplectic potentials $s: \breve{P} \subset$ $\left(\mathbb{R}^{+}\right)^{n} \rightarrow \mathbb{R}$ of the form

$$
s(x)=\frac{1}{2}\left(\sum_{i=1}^{n} x_{i} \log x_{i}+h(r)\right),
$$


where

$$
r=x_{1}+\cdots x_{n}
$$

and $\breve{P}$ will be determined in each of the particular cases that we will consider. A simple computation shows that

$$
\operatorname{Det}(S)=\frac{1+r h^{\prime \prime}(r)}{2^{n} x_{1} \cdots x_{n}} \quad \text { and } \quad S^{-1}=\left(s^{i j}=2\left(\delta_{i j} x_{i}-x_{i} x_{j} f(r)\right)\right),
$$

where $f=h^{\prime \prime} /\left(1+r h^{\prime \prime}\right)$. It then follows from (6) that the scalar curvature of the corresponding toric Kähler metric is given by

$$
S c(x)=S c(r)=2 r^{2} f^{\prime \prime}(r)+4(n+1) r f^{\prime}(r)+2 n(n+1) f(r) .
$$

The Euler-Lagrange equation defining an extremal Kähler metric can be shown to be equivalent to

$$
\frac{\partial S c}{\partial x_{j}} \equiv \text { constant, } j=1, \ldots, n,
$$

i.e. the metric is extremal if and only if its scalar curvature $S c$ is an affine function of $x$ (see [1]).

Requiring that the scalar curvature $S c \equiv S c(r)$, given by (8), is an affine function of $r$ is easily seen to be equivalent to

$$
h^{\prime \prime}(r)=-\frac{1}{r}+\frac{r^{n-1}}{r^{n}-A-B r-C r^{n+1}-D r^{n+2}},
$$

where $A, B, C, D \in \mathbb{R}$ are the 4 parameters of the family.

As shown by Calabi in [7], one can determine explicit values for the constants $A, B, C, D \in \mathbb{R}$ so that the corresponding symplectic potential, given by (7), gives rise to an extremal Kähler metric on

$$
H_{m}^{n}:=\mathbb{P}(\mathcal{O}(-m) \oplus \mathbb{C}) \longrightarrow \mathbb{P}^{n-1}, n, m \in \mathbb{N} .
$$

In our framework, this can be seen as follows. Up to a $G L(n, \mathbb{R})$ transformation, generalizing to higher dimensions the one considered in Example 3.18, $H_{m}^{n}$ is determined by a moment polytope $P_{m}^{n}(a, b) \subset \mathbb{R}^{n}$ with defining affine functions

$$
\ell_{i}(x)=x_{i}, \forall i=1, \ldots, n, \quad \ell_{n+1}(x)=\frac{1}{m}(r-a) \quad \text { and } \quad \ell_{n+2}(x)=\frac{1}{m}(b-r),
$$

where the real numbers $0<a<b$ determine the Kähler class, i.e. the cohomology class of the symplectic form $\omega_{a, b}$. Hence, if follows from Theorem 3.15 that, to determine a toric compatible complex structure on $\left(H_{m}^{n}, \omega_{a, b}\right)$, the symplectic potential has to be of the form

$$
2 s(x)=\sum_{i=1}^{n} x_{i} \log x_{i}+\frac{1}{m}((r-a) \log (r-a)+(b-r) \log (b-r))+\tilde{h}(r),
$$

where $\tilde{h}$ is smooth on $P_{m}^{n}(a, b) \subset \mathbb{R}^{n}$. Comparing (7), (10) and (11), one concludes that we must have

$$
\frac{r^{n-1}}{r^{n}-A-B r-C r^{n+1}-D r^{n+2}}=\frac{1}{m}\left(\frac{1}{r-a}+\frac{1}{b-r}\right)+R(r),
$$

where $R(r)$ is a smooth function on $P_{m}^{n}(a, b) \subset \mathbb{R}^{n}$. This gives rise to a system of 4 linear equations in the 4 unknowns $A, B, C, D \in \mathbb{R}$, which admits a unique explicit solution for any $n, m \in \mathbb{N}$ and $a, b \in \mathbb{R}$ such that $0<a<b$ (see page 285 of [7] or [22]).

Particular Cases. By construction, all Kähler metrics in Calabi's 4-parameter family are extremal. A simple computation shows that their scalar curvature is given by

$$
S c(r)=2(n+1)((2+n) D r+n C) \text {. }
$$

Hence, these metrics have

$$
\text { constant scalar curvature iff } D=0
$$

and are

$$
\text { scalar-flat iff } C=D=0 \text {. }
$$


Moreover, one can show that these metrics are

$$
\text { Kähler-Einstein iff } B=D=0
$$

and

$$
\text { Ricci-flat iff } B=C=D=0 .
$$

We will now analyse in more detail these constant scalar curvature particular cases. Note that when $A=B=C=D=0$ we have

$$
s(x)=\frac{1}{2} \sum_{i=1}^{n} x_{i} \log x_{i},
$$

which is the standard symplectic potential of the Delzant set $P=\left(\mathbb{R}_{0}^{+}\right)^{n}$ and determines the standard flat Euclidean metric on $\mathbb{R}^{2 n}$ (cf. Example 3.10).

Ricci-Flat Metrics. Assume that $B=C=D=0$ and $A=a^{n}$ with $0<a \in \mathbb{R}$. Then

$$
\begin{aligned}
h^{\prime \prime}(r) & =-\frac{1}{r}+\frac{r^{n-1}}{r^{n}-a^{n}} \\
& =-\frac{1}{r}+\frac{r^{n-1}}{(r-a) \sum_{k=1}^{n} a^{k-1} r^{n-k}} \\
& =-\frac{1}{r}+\frac{1}{n} \cdot \frac{1}{r-a}+R(r),
\end{aligned}
$$

where $R(r)$ is a smooth function on the rational Delzant set $P^{n}(a) \subset \mathbb{R}^{n}$ with defining affine functions

$$
\ell_{i}(x)=x_{i}, \forall i=1, \ldots, n, \quad \text { and } \quad \ell_{n+1}(x)=\frac{1}{n}(r-a) .
$$

The symplectic potential can be written as

$$
s(x)=\frac{1}{2}\left(\sum_{i=1}^{n} x_{i} \log x_{i}+\frac{1}{n}(r-a) \log (r-a)+\tilde{h}(r)\right),
$$

where $\tilde{h}$ is smooth on $P^{n}(a) \subset \mathbb{R}^{n}$. Hence, for each $a>0$, it defines a Ricci-flat Kähler metric on the total space of the canonical line bundle

$$
\mathcal{O}(-n) \longrightarrow \mathbb{P}^{n-1}
$$

(as before, up to a $G L(n, \mathbb{R})$ transformation, the underlying non-compact toric symplectic manifold is determined by $\left.P^{n}(a) \subset \mathbb{R}^{n}\right)$. These are the metrics constructed by Calabi in [6].

Scalar-Flat Metrics. We will now show that Calabi's family also contains the complete scalarflat Kähler metrics on the total space of the line bundles

$$
\mathcal{O}(-m) \longrightarrow \mathbb{P}^{n-1}, \forall m \in \mathbb{N},
$$

constructed by LeBrun [18] and Pedersen-Poon [21] (see also Simanca [24]).

Up to a $G L(n, \mathbb{R})$ transformation, these spaces are determined by the rational Delzant sets $P_{m}^{n}(a) \subset \mathbb{R}^{n}$, with $0<a \in \mathbb{R}$ and defining affine functions

$$
\ell_{i}(x)=x_{i}, \forall i=1, \ldots, n, \quad \text { and } \quad \ell_{n+1}(x)=\frac{1}{m}(r-a) .
$$

which means that the symplectic potential has to be of the form

$$
s(x)=\frac{1}{2}\left(\sum_{i=1}^{n} x_{i} \log x_{i}+\frac{1}{m}(r-a) \log (r-a)+\tilde{h}(r)\right),
$$

where $\tilde{h}$ is smooth on $P_{m}^{n}(a)$. This implies that

$$
h^{\prime \prime}(r)=-\frac{1}{r}+\frac{1}{m} \cdot \frac{1}{r-a}+R(r),
$$


with $R(r)$ smooth on $P_{m}^{n}(a)$. Since the scalar-flat condition is equivalent to $C=D=0$, we get that

$$
-\frac{1}{r}+\frac{r^{n-1}}{r^{n}-A-B r}=-\frac{1}{r}+\frac{1}{m} \cdot \frac{1}{r-a}+R(r) .
$$

This relation gives rise to a system of 2 linear equations in the 2 unknowns $A, B \in \mathbb{R}$, which admits a unique solution for any $n, m \in \mathbb{N}$ and $0<a \in \mathbb{R}$ :

$$
A=a^{n}(1-n+m) \text { and } B=(n-m) a^{n-1} .
$$

Note that when $m=1$ we get complete scalar-flat Kähler metrics on the total space of the line bundle

$$
\mathcal{O}(-1) \longrightarrow \mathbb{P}^{n-1},
$$

i.e. on $\mathbb{C}^{n}$ blown-up at the origin. These were originally constructed by D. Burns (at least when $n=2)$.

Fubini-Study and Bergman Metrics. Assume that $A=B=D=0$, which implies in particular that we are considering Kähler-Einstein metrics. Then

$$
\begin{aligned}
h^{\prime \prime}(r) & =-\frac{1}{r}+\frac{r^{n-1}}{r^{n}-C r^{n+1}} \\
& =-\frac{1}{r}+\frac{1}{r(1-C r)} \\
& =\frac{1}{\frac{1}{C}-r},
\end{aligned}
$$

which implies that the symplectic potential can be written as

$$
s(x)=\frac{1}{2}\left(\sum_{i=1}^{n} x_{i} \log x_{i}+\left|\frac{1}{C}-r\right| \log \left|\frac{1}{C}-r\right|\right) .
$$

When $C=1$ we recover Example 3.13, i.e. the standard complex structure and Fubini-Study metric on $\mathbb{P}^{n}$. More generally, for any $C>0$, this defines the standard complex structure and suitably scaled Fubini-Study metric on $\mathbb{P}^{n}$. The corresponding moment polytope is the simplex in $\mathbb{R}^{n}$ with defining affine functions

$$
\ell_{i}(x)=x_{i}, \forall i=1, \ldots, n, \quad \text { and } \quad \ell_{n+1}(x)=\frac{1}{C}-r .
$$

When $C<0$ it follows from Theorem 3.15 that the above symplectic potential determines a toric compatible complex structure $J_{C}$ on the toric symplectic manifold $\left(\mathbb{R}^{2 n}, \omega_{\text {st }}\right)$ with Delzant set $P=\left(\mathbb{R}_{0}^{+}\right)^{n} \subset \mathbb{R}^{n}$. The corresponding Kähler metric is a $U(n)$-invariant Kähler-Einstein metric of negative scalar curvature on the complex manifold $\left(\mathbb{R}^{2 n}, J_{C}\right)$. Using the holomorphic coordinates given by (4), one easily concludes that $\left(\mathbb{R}^{2 n}, J_{C}\right)$ is biholomorphic to a ball $B \subset \mathbb{C}^{n}$, which implies in particular that $J_{C}$ is not complete. Moreover, the Kähler metric $\langle\cdot, \cdot\rangle_{C}:=\omega_{\mathrm{st}}\left(\cdot, J_{C} \cdot\right)$ is, in fact, the well-known and complete Bergman metric.

Other Kähler-Einstein Metrics. We will now show that Calabi's family also contains the complete Kähler-Einstein metrics with negative scalar curvature on the total space of the open disc bundles

$$
\mathcal{D}(-m) \subset \mathcal{O}(-m) \longrightarrow \mathbb{P}^{n-1}, \forall n<m \in \mathbb{N},
$$

constructed by Pedersen-Poon [21].

As toric symplectic manifolds, and up to a $G L(n, \mathbb{R})$ transformation, these spaces are again determined by the rational Delzant sets $P_{m}^{n}(a) \subset \mathbb{R}^{n}$ with defining affine functions

$$
\ell_{i}(x)=x_{i}, \forall i=1, \ldots, n, \quad \text { and } \quad \ell_{n+1}(x)=\frac{1}{m}(r-a) .
$$

which means that the symplectic potential has to be of the form

$$
s(x)=\frac{1}{2}\left(\sum_{i=1}^{n} x_{i} \log x_{i}+\frac{1}{m}(r-a) \log (r-a)+\tilde{h}(r)\right),
$$


where $\tilde{h}$ is smooth on $P_{m}^{n}(a)$. This implies that

$$
h^{\prime \prime}(r)=-\frac{1}{r}+\frac{1}{m} \cdot \frac{1}{r-a}+R(r),
$$

with $R(r)$ smooth on $P_{m}^{n}(a)$. Since the Kähler-Einstein condition is equivalent to $B=D=0$, we get that

$$
-\frac{1}{r}+\frac{r^{n-1}}{r^{n}-A-C r^{n+1}}=-\frac{1}{r}+\frac{1}{m} \cdot \frac{1}{r-a}+R(r) .
$$

This relation gives rise to a system of 2 linear equations in the 2 unknowns $A, C \in \mathbb{R}$, which admits a unique solution for any $n<m \in \mathbb{N}$ and $0<a \in \mathbb{R}$ :

$$
A=\frac{(m+1) a^{n}}{n+1}>0 \text { and } C=\frac{n-m}{(n+1) a}<0 .
$$

As remarked by Pedersen-Poon, these metrics are a superposition of Calabi's Ricci-flat metrics $(A>0)$ and Bergman metrics $(C<0)$. The analogous superposition of Calabi's Ricci-flat metrics $(A>0)$ with Fubini-Study metrics $(C>0)$ gives rise to Kähler-Einstein metrics on the projectivization of the above line bundles, with cone-like singularities in the normal directions to the zero and infinity sections. As explained in [4], these metrics are directly related to the smooth Sasaki-Einstein metrics constructed in 2004 by Gauntlett-Martelli-Sparks-Waldram [13, 14].

Other Constant Scalar Curvature Metrics. We will now show that Calabi's family also contains the complete constant negative scalar curvature Kähler metrics on the total space of the open disc bundles

constructed again by Pedersen-Poon [21].

$$
\mathcal{D}(-m) \subset \mathcal{O}(-m) \longrightarrow \mathbb{P}^{n-1}, \forall n, m \in \mathbb{N},
$$

As before, we are interested in the rational Delzant sets $P_{m}^{n}(a) \subset \mathbb{R}^{n}$ and symplectic potentials of the form

$$
s(x)=\frac{1}{2}\left(\sum_{i=1}^{n} x_{i} \log x_{i}+\frac{1}{m}(r-a) \log (r-a)+\tilde{h}(r)\right),
$$

with $\tilde{h}$ smooth on $P_{m}^{n}(a)$. Assuming $D=0$ and $C=-1$, i.e. $S c=-2 n(n+1)$, this implies that

$$
-\frac{1}{r}+\frac{r^{n-1}}{r^{n}-A-B r+r^{n+1}}=-\frac{1}{r}+\frac{1}{m} \cdot \frac{1}{r-a}+R(r),
$$

with $R(r)$ smooth on $P_{m}^{n}(a)$. This relation gives rise to a system of 2 linear equations in the 2 unknowns $A, B \in \mathbb{R}$, which admits a unique solution for any $n, m \in \mathbb{N}$ and $0<a \in \mathbb{R}$ :

$$
A=(m-n+(1-n) a) a^{n} \quad \text { and } \quad B=(n-m+1+n a) a^{n-1} .
$$

\section{REFERENCES}

[1] Abreu M., Kähler Geometry of Toric Varieties and Extremal Metrics, Internat. J. Math. 9 (1998) 641-651.

[2] Abreu M., Kähler Geometry of Toric Manifolds in Symplectic Coordinates, In: Symplectic and Contact Topology: Interactions and Perspectives, Y.Eliashberg, B.Khesin and F.Lalonde (Eds), Fields Institute Communications 35, American Mathematical Society 2003, pp 1-24.

[3] Abreu M., Kähler Metrics on Toric Orbifolds, J. Differential Geom. 58 (2001) 151-187.

[4] Abreu M., Kähler-Sasaki Geometry of Toric Symplectic Cones in Action-Angle Coordinates, to appear in the proceedings of the Geometry Summer School, Lisbon, July 13-17, 2009.

[5] Atiyah M., Convexity and Commuting Hamiltonians, Bull. London Math. Soc. 14 (1982) 1-15.

[6] Calabi E., Métriques Kähleriennes et Fibrés Holomorphes, Ann. Sci. Ec. Norm. Sup. 12 (1979) $269-294$.

[7] Calabi E., Extremal Kähler Metrics, In: Seminar on Differential Geometry, S.-T. Yau (Ed), Annals of Math. Studies 102, Princeton Univ. Press 1982, pp 259-290.

[8] Calabi E., Extremal Kähler Metrics II, In: Differential Geometry and Complex Analysis, I. Chavel and H. Farkas (Eds), Springer-Verlag 1985, pp 95-114.

[9] Calderbank D., David L. and Gauduchon P., The Guillemin Formula and Kähler Metrics on Toric Symplectic Manifolds, J. Symplectic Geom. 1 (2003) 767-784.

[10] Delzant T., Hamiltoniens Périodiques et Images Convexes de l'Application Moment, Bull. Soc. Math. France 116 (1988) 315-339.

[11] Donaldson S., Remarks on Gauge Theory, Complex Geometry and 4-manifold Topology, In: Fields Medallists' Lectures, M. Atiyah and D. Iagolnitzer (Eds), World Scientific 1997, pp 384-403. 
[12] Donaldson S., Scalar Curvature and Stability of Toric Varieties, J. Differential Geom. 62 (2002) 289-349.

[13] Gauntlett J., Martelli D., Sparks J. and Waldram D., Sasaki-Einstein Metrics on $S^{2} \times S^{3}$, Adv. Theor. Math. Phys. 8 (2004) 711-734.

[14] Gauntlett J., Martelli D., Sparks J. and Waldram D., A New Infinite Class of Sasaki-Einstein Manifolds, Adv. Theor. Math. Phys. 8 (2004) 987-1000.

[15] Guillemin V., Kähler Structures on Toric Varieties, J. Differential Geometry 40 (1994) 285-309.

[16] Guillemin V. and Sternberg S., Convexity Properties of the Moment Mapping, Invent. Math. 67 (1982) 491513.

[17] Karshon Y. and Lerman E., Non-Compact Symplectic Toric Manifolds, arXiv:0907.2891.

[18] LeBrun C., Counter-Examples to the Generalized Positive Action Conjecture, Comm. Math. Phys. 118 (1988) 591-596.

[19] Lerman E. and Tolman S., Hamiltonian Torus Actions on Symplectic Orbifolds and Toric Varieties, Trans. Amer. Math. Soc. 349 (1997) 4201-4230.

[20] Martelli D., Sparks J. and Yau S.-T., The Geometric Dual of a-maximisation for Toric Sasaki-Einstein Manifolds, Comm. Math. Phys. 268 (2006) 39-65.

[21] Pedersen H. and Poon Y., Hamiltonian Constructions of Kähler-Einstein Metrics and Kähler Metrics of Constant Scalar Curvature, Comm. Math. Phys. 136 (1991) 309-326.

[22] Raza A., An Application of Guillemin-Abreu Theory to a Non-Abelian Group Action, Differential Geom. Appl. 25 (2007) 266-276.

[23] Siegel C., Symplectic Geometry, Amer. J. Math. 65 (1943) 1-86.

[24] Simanca S., Kähler Metrics of Constant Scalar Curvature on Bundles Over $\mathbb{C P}^{n-1}$, Math. Ann. 291 (1991) 239-246.

Centro de Análise Matemática, Geometria e Sistemas Dinâmicos, Departamento de Matemática, Instituto Superior Técnico, Av. Rovisco Pais, 1049-001 Lisboa, Portugal

E-mail address: mabreu@math.ist.utl.pt 